\title{
Challenge of 2D representation of 3D objects in architectural and heritage studies. In search of gaze patterns similarities
}

Marta Rusnak ( $\sim$ marta.rusnak@pwr.edu.pl)

Wrocław University of Science and Technology

\section{Research Article}

Keywords:

Posted Date: March 1st, 2022

DOI: https://doi.org/10.21203/rs.3.rs-1371709/v1

License: @ (i) This work is licensed under a Creative Commons Attribution 4.0 International License. Read Full License 


\section{Abstract}

This paper describes the results of eye-tracking registrations using spherical stimuli related to the so-called longitudinal church effect and compared it to those obtained by using a stationary eye-tracker and pictures shown on a computer screen in previous studies. The data collected from nearly 120 viewers allowed to investigate the effect of length of the nave on concentration on the altar and its role in supporting the ritual. An important aspect of this study is that it compares three pairs of stimuli and their relationships, rather than just two examples. The experiment with flat images in many respects gave results like those obtained in virtual reality. Even though the values on which the conclusions were based differed, 11 out of 14 analyzed aspects were confirmed. An important difference observed in VR was a noticeable decrease in the average duration of fixation. Thanks to the presented comparison it can be assumed that there is no need to involve VR technology if the studied phenomena are simple and the most important elements of linear compositions are visible in a single glance. If eye-tracking tests conducted with stationary eye-trackers do not have any other flaws, the conclusions drawn from them can be trusted.

\section{Introduction}

Using eye-tracking in neuroaesthetics research allowed us to get a closer look at the issue as described by numerous historians of art, conservators, and architects of the past ${ }^{1,2}$. Experimental recognition of an aesthetic experience raised many questions ${ }^{3,4}$. The combination of an eye-tracker and VR (Virtual Reality) goggles has opened up new perspectives for scientists dealing with perception. This new research environment has made it necessary to clarify any unclear points, especially those concerning validity of research done on three-dimensional objects presented as flat images. A visualization displayed on a screen as a flat image is a considerable simplification of the same object seen in reality or presented in the form of a spherical image ${ }^{5}$. While eye-tracking research on paintings ${ }^{6}$ or pictures displayed on a screen 7,8 provokes aesthetics questions and controversies concerning visual attention ${ }^{9}$ similar research may be more doubtful if 3D objects - such as sculptures, landscapes or buildings - are presented as $2 \mathrm{D}$ stimuli ${ }^{10}$. When scientists choose how the given object is presented (such as size of a screen, the perspective from which it is seen, its brightness or contrast levels) they cannot be sure how such simplification affects the results of the conducted research. Similarly, research dealing with hypothetical issues - e.g., related to nonexistent interiors or alternative versions of an existing structure - simply cannot be done in real-life surroundings. Due to technological limitations, scientists had to use visualizations that would then be displayed on a screen. Nowadays, when we have portable eye-trackers making it possible to display augmented reality stimuli or eye-trackers applicable in VR environment ${ }^{11}$, should scientists dealing with the perception of material heritage give up on simplifying the cognitive scenario ${ }^{12}$ ? Should they stop using stationary eye trackers? Some conducted studies $^{13,14}$ make it extremely difficult to acknowledge flat representation of architecture as controllable or categorizable stimuli ${ }^{15,16}$ like paintings. This motivated to answer the question - did the research involving flat displays - films, photographs, photomontages, and visualizations of architecture- yield false results? Similar questions, regarding the influence of the environment on the conducted research, asked Gulhan, Durant and Zenker. They compared scan paths and gaze allocation while perceiving the real museum exposition and the virtual equivalent ${ }^{17}$. Other researchers, guided by Prof. Dong, were looking for differences and similarities in pathfinding between reality and VR experience ${ }^{18}$. The experiment is different with regard to the aforementioned studies because not only two situations are compared, but three pairs of stimuli as well as the relationships between them. The new study was meant to analyze a similar problem, how interiors of gothic cathedrals are perceived on flat and spherical images?

Research relating to Gothic architecture makes it possible to state that constructors of medieval cathedrals aimed at a harmonious combination of form, function and message ${ }^{19-21}$. It is believed that alterations in the proportions of these buildings and the appearance of the details in them were directly related to the mystical significance of such spaces. The same can be said about the altar. Presbytery, closing the axis of a church, is the most important part of such a structure. The shape of the cathedral's interior should facilitate focusing one's eyesight on that great closure, making it easier for the 
congregation to concentrate on the religious ritual taking place there. The so-called longitudinal church effect ${ }^{22}$ discovered that the evolution in thinking about the proportions of sacral buildings led medieval builders to deliberately lengthen the naves of their cathedrals. As a result, those entering such a building were able to better comprehend the metaphysical hierarchy of the interior's layout. In experiment, longer nave facilitated the participants to better concentrate on the altar and its immediate surroundings; they also spent less time looking to the sides and the way in which they moved their eyes was less dynamic. The conclusions of mentioned research indicated a need for further methodological experiments in the field of eye-tracking tests relating to the perception of architectural heritage. In 2016, what was available was research using flat visualizations of cathedral interiors examined with a stationary eye tracker or experiments done in a real-life situation with a portable eye tracker. As of today, eye tracking devices and the software that accompanies them permit experiments in virtual reality environments. Undertaking the same topic using a more advanced tool might be not only interesting but scientifically necessary.

Research centered on stimuli displayed in VR makes use of the same mechanisms as portable eye trackers. The difference is that fixations are not marked on a film recorded by a camera aimed at whatever the experiment's participant is looking at but registered in relation to objects seen by such a person in their VR goggles ${ }^{23}$. VR eye tracking is becoming increasingly popular in research devoted to human-computer interactions. In the last three years a considerable number of tests were connected to pedagogy ${ }^{24}$, education ${ }^{25}$ and linguistics ${ }^{26}$. Such research was also applied in the fields of marketing ${ }^{27}$ as well as to identify dangers in buildings ${ }^{28}$ and at construction sites ${ }^{29}$. Eye trackers combined with VR goggles remain a novelty, but such a combination has already been applied in research into historical areas ${ }^{30}$, landscape ${ }^{31}$ and cityscape protection $^{32}$. One should not neglect the variety of ways in which other types of eye trackers have been used in studies over cultural heritage. For example, they have been used to analyze perception of paintings ${ }^{33}$ behaviors of museum visitors ${ }^{34}$, perception of historical monuments ${ }^{35}$, interiors ${ }^{36}$, natural ${ }^{37}$ and urbanized environments ${ }^{38}$. perceived by different users ${ }^{39}$. It is not difficult to notice the growing interest in the so-called Neuro-architecture ${ }^{40}$ eye-tracking architecture belongs to this dynamically developing field ${ }^{41}$. It is more than likely that a large portion of such tests will be done in VR in near future. Therefore there is a strong scientific motivation to have a better look at the pros and cons of this kind of research.

The most basic aim of the research was to compare the data presented in the aforementioned paper ${ }^{22}$, where the experiment's participants looked passively at flat images, with the results obtained in the new experiment employing VR technology, which allowed movement. The idea was to verify the way people respond visually to interiors of churches of different proportions as well as to scrutinize whether the longitudinal church effect exists. The author intended to see whether her previous decision to use a stationary eye tracker was a valid scientific move. Such a comparison also makes it possible to have a better look at the upsides and downsides of two research environments. The author feels that it is important since eye trackers are quickly gaining popularity as research tools for studying people's perception. The rapid growth of interest in this field is testified to by the EBSCO database data that Sylwester Białowąs and Adrianna Szyszka wrote about ${ }^{42}$. Pioneering implementations of new technologies is often characterized by a lack of critical methodological approach $^{43}$. The quality of data depends on multiple factors, one of which is the system in which the research is conducted $^{44}$. It should be noticed that other scholars have expressed a similar need for a discussion on advantages and disadvantages of various environments used for gathering behavioral data. For instance, what has been studied is the difference in how people perceive their surroundings when they are having a stroll through the city and just watching a recording from such a walk ${ }^{45}$ and several fascinating differences and similarities were observed. Other research focused on the behaviors of people making purchases in different kinds of virtual space ${ }^{46}$. One might also wonder whether the results of comparison of passive and active perception will match the tendencies described by Haskins, Mentch, Botch et al. according to which the observations that allow head movement "increased attention to semantically meaningful scene regions, suggesting more exploratory, information-seeking gaze behavior" 47 . If it works that way, those viewers who are allowed to move their heads should spend most time looking at the presbytery and the vaults ${ }^{22}$. On this level, it is also possible to raise the question, whether the visual behavior will change in the same way as has been observed in the 
museum when comparing scan paths in VR and reality. Would there be a tendency to switch focus rapidly if the average fixation duration decreased in $\mathrm{VR}^{17}$ ?

\section{Methods}

Methodology of the VR experiment was as similar as possible to that applied to the previous experiment involving stationary eye tracker. The most important aspects were connected with selection of participants, presentation of stimuli, the number of additional displayed images.

\section{Research tools and software}

The set used in the experiment consisted of HTC Vive goggles and Tobii Pro Integration eye tracker. The virtual research space had been orientated using SteamVR Base Station 2.0. The eye-tracking registration was done on both eyes with a frequency of $120 \mathrm{~Hz}$, accuracy of $0.5^{\circ}$ and the trackable field of view was $110^{\circ} 48$. Additional spherical images were produced using Samsung Gear 360. Tobii Pro Lab 360VR software was used in the experiment. The model of the cathedral and its versions, and final stereoscopic panoramas were developed in Blender, version 2.68. Most of the materials come from free online sources. The remaining textures were created in Adobe Substance Painter.

\section{Participants}

In accordance with the conclusions drawn from previous studies, the number of participants was increased. 150 people were invited to take part. Analogically to the previous research, the participants were only accepted if they had no education related to the field of research (i.e., historians of art, architects, city planners, conservators were all excluded). Another excluded group - on the basis of the information provided in the application form - were those who had already taken part in the previous research since they might remember the displayed stimuli and there would be no way of verifying to what extent that affected the results. All volunteers were Polish adults under 65 (Figure S1), living within the Wrocław agglomeration. The preliminary survey was also intended to exclude people with diagnosed major vision impairments those with problems with color vision, strabismus, astigmatism, cataract, impaired eye mobility. Participants could use their contact lenses. From the scientific perspective, it also seemed crucial that the participants have a similar approach to the employed VR environment. None of the participants possessed such a device. $87 \%$ of volunteers had had no experience with VR goggles. The other $13 \%$ had had some experience with VR, but of a very limited sort - only for 5-10 minutes and not more than twice in their lifetime. Therefore it is reasonable to claim that the participants constituted a fairly homogeneous group of people unfamiliar with the applied scientific equipment. The way participants were familiarized with a new situation and how personalization of settings was conducted is described in supplementary materials (see Personalization of settings and making the participants familiar with a new situation). All experimental protocols were approved by authorities of the Faculty of Architecture and the Research Ethics Committee at Wrocław University of Science and Technology. All methods were performed in accordance with the ethical guidelines and regulations of the Declaration of Helsinki. Informed consent was obtained from all participants.

\section{Design}

\section{Used spherical stimuli}

The inability to move makes the immersion of the presented environment smaller ${ }^{49}$. However to allow comparison with previously performed studies, the participants were not allowed to move. For this reason, only stationary spherical image was used in research (Fig. 1). The ratios, the location and size of used details, materials, colors, contrasts, or intensity and angle at which the light is cast in the interior was identical to previous work ${ }^{22}$. Just like before three stimuli of varied nave length were generated. The ratios of the prepared visualizations were based on real buildings of this kind. The images were 
named as follows: AS - cathedral with a short nave, BS - cathedral with a medium-length nave, and CS - cathedral with a long nave (Fig. 1).

\section{Spherical images and flat images}

Perhaps the most striking difference between the two experiments is the scope of the image that the participants of the VR test were exposed to. The use of a spherical image made it possible to turn one's head and look in all directions. Those who took part in the original test saw only a predetermined fragment of the interior. For the purpose of this study, this AOI was named "Old Research". The range of the architectural detail visible in the first test was marked with a red frame (Fig. 1). It might seem that the participants exposed to a flat image saw less than one-tenth of what the participants of the VR test were shown. This illusion stems from the nature of a flattened spherical image, which additionally enlarges the areas located close to the vantage point by strongly bending all nearby horizontal lines and surfaces. The same distortion is responsible for the fact that the originally square image here has a rounded top and bottom. An aspect where achieving homogeneous conditions proved difficult was the attempt to achieve the same balance of color, contrast and brightness on both the computer screen and the projector in the VR goggles. Finally, the settings - once deemed a satisfactory approximation of those applied in the original test - were not altered during the experiment.

\section{Procedure}

The experiment, just like the original research, included nine auxiliary images (Figures S2-S10). The presented material consisted of spherical photos taken inside Wrocław churches. Each image, including the photos and the visualizations, was prepared so that the presbytery in each case would be located in the same spot, on the axis, in front of the viewer (Fig. 2). Just like in the previous research it was important to make it impossible for the participants to use their sort-term memory while comparing churches with different lengths of nave, so each participant's set of images included only one of the images under discussion (AS-short or BS-medium-length or CS-long). Therefore three sets of stimuli had been prepared. Each included instructional boards informing the participants about the rules of the experiment and allowing an individual calibration of the eye tracker, one of the three analyzed images and nine auxiliary images in the form of the aforementioned spherical photos. Analogically to the original experiment, the participants were given the same false task they were supposed to identify those displayed buildings which, in their opinion, were located in Wrocław. This was meant to incite a homogeneous cognitive intention among the participants ${ }^{50}$ when they looked at a new, uniquely unfamiliar interior. Since it was expected that the VR technology would be a fairly new experience for the majority of the participants, it was decided that the prepared visualizations would not be shown as one of the first three stimuli in a set. Other images were displayed in random order.

During that previous test, a trigger point function of the BeGaze (SMI) software was used, which allowed automatic display of an image once the participant looked at a specific predefined zone on additional bord. In Tobii ProLab such a feature was not available. All visual stimuli were separated from one another by means of an additional spherical image. When participants looked at red dot the decision to display the next image had to be made by the person supervising the entire process.

The time span of registration that had been calculated for the original study was kept unchanged and amounted to 8 seconds. However, this proved insufficient for the participants - 5 out of 7 volunteers, invited for preliminary tests and not included in the group undergoing analysis, expressed the need to spend more time looking at the images. It was suggested that the brevity of the display made the experience uncomfortable. That is why the duration of a single display was increased to 16 seconds, but only the first 8 were taken into consideration and used in the comparison.

\section{Preparation of the room}

Looking at a spherical image in VR did not allow movement within the displayed stimuli therefore the participants were asked to sit, just like in the study involving a stationary eye tracker. A change in the position of the participant's body would 
require a change in the height of the camera and, as a result, a change in the perspective and that might have an adverse effect on the experiment. The room was quiet and allowed to dim the light. The positions of the participant's seat and the VR base unit were marked on the room's floor so as to make it possible to check and, if necessary, correct accidental shifts of the equipment (Fig. 2). It was important since any shifts in the position of the equipment would affect the orientation of the stimuli in space, making the direction in which the participant is initially looking inconsistent with the axes of the displayed interiors.

\section{Results}

Despite the fact that it is detached from real space research evaluate one case study. When comparing the test to other eye-tracking tests, mentioned in the introduction, the final data from 117 people is satisfactory. From a traditional point of view, the sample is small so the research has potentially low power, which minimizes the likelihood of reproducibility.

This section of the article describes only newly collected data. Numerical data were generated using Tobii Pro Lab ${ }^{51}$. Thanks to the data on all registrations, collective numerical reports were prepared. Four main types of eye-tracking variables were analyzed: fixation count, visitors number, average fixation duration, total fixation duration. Heat maps were generated automatically in Tobii Pro Lab. XLS reports have been processed in Microsoft Excel. In discussion section the data will be compared with the results of the previous experiment.

Finally, biometric data was gathered correctly from 40 people who had been shown stimulus AS, 40 people who had been shown stimulus BS, and 37 people who had been shown stimulus CS. That means that for various reasons $22 \%$ of registrations were deemed unusable for the purposes of the analysis. The reasons why some volunteers resigned from participating as well as the way the researchers verified the correctness of the data-collecting process and the quality of the eye-tracking registration is described in the supplementary materials (see Reasons for resignation from the experiment and ways of verifying the manner of collecting data and the quality of registrations).

Due to the change in equipment and the environment of the experiment, more Areas of Interest were analyzed than during the previous study ${ }^{22}$. The way people looked at the spherical images was analyzed by dividing each of them into ten AOls. Their location and names can be seen in Fig. 1. In the frame located in the center one may find AOls with a caption saying "old" - that is because they correspond to the AOls used in the flat images in the first study. All these AOls can be summed up into one area named Old Research. Five more AOls, which are placed over the parts of the image that had not been visible to those exposed to the flat stimuli, were captioned with names starting with "new".

The analysis began with a comparison of heat maps (Figure S11). It appears that the observers of the church with the longest nave paid the most attention to the presbytery and the fragment shown in the previous study. As opposed to AS and $A B$, the heatmap of the CS example has three, not two, spots of intense color. An additional point is located within the vault.

Fixation report was done for the entire spherical image but also for all five Old and five New Research AOl's (Table 1). The number of fixations performed on entire stimuli AS, BS and CS is not significantly different. Much more fixations were done on the "Old AOI". The number of fixations performed within the five new AOls decreases with the process of lengthening the interior. The values for the fields visible in the previous test increase as the cathedral is extended. 
Table 1

Fixation count for entire spherical image and two mail AOls.

\begin{tabular}{|llll|}
\hline $\begin{array}{l}\text { Name of } \\
\text { 3D stimulus }\end{array}$ & $\begin{array}{l}\text { Entire spherical } \\
\text { image }\end{array}$ & $\begin{array}{l}\text { Old Research AOI } \\
\text { the part of the image visible in the } \\
\text { previous study }\end{array}$ & $\begin{array}{l}\text { New Research AOI } \\
\text { parts of the image not visible in the } \\
\text { previous study }\end{array}$ \\
\hline $\begin{array}{l}\text { Short cathedral AS } \\
\begin{array}{l}\text { Medium-length } \\
\text { cathedral BS }\end{array}\end{array}$ & $23.3 \mathrm{fix}$ & $16.3 \mathrm{fix}$ & $7,0 \mathrm{fix}$ \\
\hline Long cathedral CS & $23.6 \mathrm{fix}$ & $16.9 \mathrm{fix}$ & $6,9 \mathrm{fix}$ \\
\hline
\end{tabular}

In order to be able to describe the interest in particular parts of the stimulus, we can look at how many people looked at them. Table 2 lists all 10 AOls analyzed and their values. When viewing the data, it is important to consider the different numbers of participants who were shown examples. Therefore the numbers are additionally shown in percentages.

Inspection of the first part of the table, it is apparent that the values presented in five columns represent a single trend. The largest difference in the number of observers is in the AOI Old vaults. For AOI Flor Old, AOI Right Nawe New and AOI Vaults New the size of a group is descending. The number of people watching the presbytery is slightly increasing.

Table 2

Summary of recorded values for $10 \mathrm{AOIs}$ determined for AS, BS and CS: 1. Visitors number, 2. Avradge fixation time, 3. Total visit duration. AS - Short cathedral, BS - Medium-length cathedral, CS - Long cathedral.

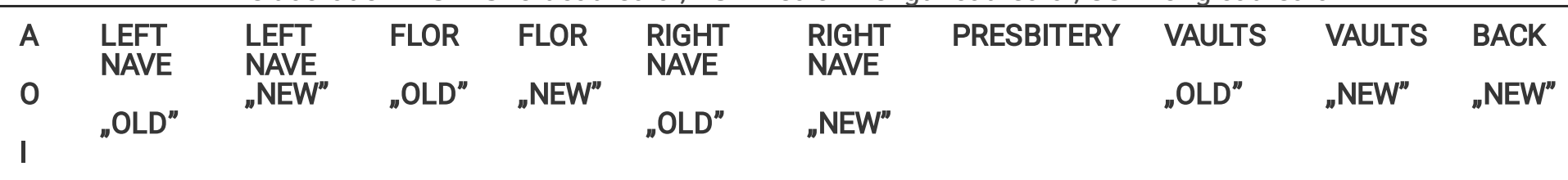

\section{Visitors number [people]}

$\begin{array}{lllllllllll}\text { AS } & 39 / 95 \% & 17 / 40 \% & 6 / 15 \% & 6 / 15 \% & 37 / 92 \% & 34 / 80 \% & 38 / 95 \% & 20 / 50 \% & 4 / 10 \% & 5 / 13 \% \\ \text { BS } & 39 / 97 \% & 17 / 40 \% & 3 / 8 \% & 6 / 15 \% & 40 / 100 \% & 31 / 78 \% & 39 / 97 \% & 36 / 90 \% & 1 / 3 \% & 0 \% \\ \text { CS } & 31 / 84 \% & 12 / 32 \% & 0 \% & 4 / 11 \% & 35 / 96 \% & 19 / 51 \% & 37 / 100 \% & 37 / 100 \% & 0 \% & 0 \%\end{array}$

\section{Average fixation duration [s]}

$\begin{array}{lllllllllll}\text { AS } & 0,163 & 0,101 & 0,007 & 0,120 & 0,167 & 0,115 & 0,170 & 0,112 & 0,09 & 0,131 \\ \text { BS } & 0,156 & 0,103 & 0,110 & 0,98 & 0,162 & 0,118 & 0,180 & 0,121 & 0,00 & 0,109 \\ \text { CS } & 0,153 & 0,075 & 0,00 & 0,00 & 0,141 & 0,112 & 0,207 & 0,133 & 0,00 & 0,00\end{array}$

3. Total visit duration [s]

\begin{tabular}{llllllllllll|} 
AS & 1,45 & 0,84 & 0,21 & 0,12 & 1,25 & 1,19 & 1,15 & 0,76 & 0,18 & 0,13 \\
BS & 1,01 & 0,78 & 0,23 & 0,10 & 1,34 & 1,01 & 1,04 & 1,18 & 0,09 & 0,13 \\
CS & 1,18 & 0,46 & 0,00 & 0,10 & 1,49 & 0,98 & 0,93 & 1,54 & 0,00 & 0,00 \\
\hline
\end{tabular}

Another analyzed parameter is Average fixation duration. The number of fixations is almost identical when considering data on entire pictures. The results are between $130 \mathrm{~ms}$ and $140 \mathrm{~ms}$ ( $\mathrm{AS}=130 \mathrm{~ms}, \mathrm{BS}=136 \mathrm{~ms}, \mathrm{CS}=139 \mathrm{~ms}$ ). All three examples of presbytery zone have the longest-lasting fixations and for CS average fixation duration exceeded the value of $200 \mathrm{~ms}$. This outstanding value demonstrates the "magnetism" of this small element. 
The last analyzed feature is Total visit duration. By far the largest difference in value was recorded for the AOI Vaults "Old". Observers of the long CS example spend twice as much time looking at the shortest vaults as they spend looking at the long vaults. Attention time also increases for AOI Right Nave "Old". The clearly opposite tendency toward focusing on both sides of the aisle during the previous experiment seems quite odd. The feature of AOI Flor "New" field turned out to be unique because it shows almost no differentiation in visit duration.

\section{Discussion}

Both studies have many aspects that need to be compared. To begin with, the results for the overall parameters computed for three spherical and three flat stimuli are compared. This general analysis confirms the accuracy of the visual analysis scoping of the previous study, thus allowing for furthermore in-depth considerations. In response to a large number of presented AOls, it was decided to create three schemes based on homogeneous rules. On this basis, the results that confirm the existence of the tunnel church and those that contradict previous observations will be discussed. Illustrations will also allow for an analysis of individual AIOs. The second stage of the introductory process will involve analyzing the number of people who looked up, down, or back. Observation of the number of people looking at a specific zone will allow determining how the changing proportions of the interior influenced the viewers. It was decided to pay special attention to the presbytery zone and the vaults, since these elements were deemed the most cognitively valuable in the previous study.

One of the primary ideas behind doing research on the same topic again was to check the correctness of the methodology used previously, including the author's choice of how much of the interior was shown in the original stimuli. Should the participants of the VR experiment look for a very limited time at this area and instead find other parts of the building more attractive, it might suggest that some errors were made when making assumptions about either the previous research or the current one. In that case it would be impossible to make a credible comparison of the results obtained during those two experiments. A general analysis was therefore done for the Old Research AOI. The value that perhaps best testifies to one's cognitive engagement is the total visit duration calculated for all the AOls visible in the previous research. The average value of this parameter amounted to $4.70 \mathrm{~s}$, which is almost $59 \%$ of the registration span, for stimulus AS; $4.14 \mathrm{~s}$, which is $52 \%$ of registration span, for BS; and $5.14 \mathrm{~s}$, which is slightly over $64 \%$ of registration span for CS. What is interesting, an average of nearly $3 / 4$ of the participants' fixations took place within the area presented in the previous research (70.175.9\%) (Table S1). That means that fixations within the "Old Research" AOI were focused and that the participants made few fixations when looking to the sides or to the back. If those points of focus were more evenly distributed, one would be entitled to assume that no part of the interior drew attention in particular. These results suggest that the "Old Research" AOI really did include the most important architectural elements in such a religious building as far as the impression made on an observer is concerned. This allows a further, more detailed analysis and comparison of the data obtained using a VR headset and those acquired by means of a stationary eye tracker.

Despite the widening of the field of view, the visual activity of viewers decreased ${ }^{39}$. There was little difference in the average number of fixations registered for participants looking at different stimuli $(A S=23.3 \mathrm{fix}, \mathrm{BS}=23.6 \mathrm{fix}, \mathrm{CS}=23.7$ fix). Similarities in the engagement of different participants - a similar number of fixations (Table 1) may be linked with the fact that for most participants the act of using VR goggles themselves was moderately stressful.

A significant change should be noted average duration of a fixation for the entire image $(A S=130 \mathrm{~ms}$ vs $A=290 \mathrm{~ms}, B S=$ $136 \mathrm{~ms}$ vs $B=263 \mathrm{~ms}, C S=139 \mathrm{~ms}$ vs $C=271 \mathrm{~ms}$ ). The average duration of fixations recorded on the screen and in the VR environment decreased twice (Fig. 3). A similar trend shortening the duration from cognitive fixation into express fixations ${ }^{52}$ in VR was noted by Gulhan, Duraant \& Zenker ${ }^{17}$. But what is important that the change is more significant.

Subsequent comparisons are made for single AOIs. In order to facilitate the comparison of the data gathered in both experiments, the names of the stimuli are expanded with distinctions of length: short cathedral AS, medium-length cathedral BS, long cathedral CS. A graphic representation, which depicts all the AOls for all three new spherical stimuli and 
three flat stimuli from old research was prepared to allow a clear comparison of a large portion of numerical data. The upper part shows the schematics for the interior with a short nave (A and AS), followed by the interiors with a mediumlength nave (B and BS), while the longest ( $C$ and $C S$ ) are displayed at the bottom. Such graph was filled in with values corresponding to the given analyzed aspect: number of observers (Fig. 4), average fixation duration (Fig. 5), total visit duration (Fig. 6). Values inserted in the middle section were compared with the schemata shown above and below. The range of the difference influenced the color-coding of the particular $\mathrm{AOI}$, as determined in the attached legend. When the values were equal, the $\mathrm{AOI}$ was cream-colored. When the analysis showed a lower value it was colored red, and when an increase was noticed it was colored green. In either case, the intensity of the color-coding corresponds with the range of the observed difference.

The volunteers were able to make a series of movements looking up, down, to the back or to the sides. Changes in such activity may be tracked by analyzing the number of people looking at particular AOls (Fig. 4) - which is discussed in the following parts of this paper.

Figure 4 shows the combination of the three schemata and also includes the number of observers for each AOI. The upper part shows green coloring of most newly added AOIs in stimulus AS. According to the legend that suggests an increased movement of the participants. The new AOls in the bottom part of the figure, which shows the data for stimulus CS, is dominated with red color. It is very easy to notice the gradual reduction of the number of participants looking in other directions than forwards when comparing those exposed to stimuli AS, BS, and CS. The new study confirms previous findings. The vault and the presbytery, for example, were most willingly observed by people looking at interior $\mathrm{C}$. One can easily notice that the number of those looking to the sides decreased along with an increase in the length of the nave. The fragment of the image within the New Right Aisle AOI was looked at by 34 out of 40 participants exposed to stimulus AS, 31 out of 40 for BS, and only 19 out of 37 for CS. Many more people neglected looking to the left, to the Left Nave "New" AOI. In case of both AS and BS it was 17 out of 40 volunteers, but for stimulus CS it was as few as 12 out of 37 people. It is plain to see that the longest nave discouraged the observers from looking to the sides of the spherical image. People observing 2D images on a screen also looked sideways more when the church was shorter and less when its nave was lengthened (Fig. 4). It has to be pointed out that there were relatively few of those who looked to the back of the spherical image. The number amounted to 5 out of 40 and 4 out of 40 people for stimuli AS and BS respectively. Here one could observe the same tendency as with looking to the sides - the longer the nave, the smaller the need to divert one's gaze from the central part of the image. That is perhaps why not even one out of 37 people exposed to CS looked at the back of the image during the 8 seconds that were recorded and analyzed (Fig. 4). Similarly to the results of an old research, the longer the nave became, the fewer people chose to look upwards at the "new vaults". The visitors count for the stimuli proved to be as follows: $A S=10 \%$ (4/40 people), $B S=2.5 \%$ ( $1 / 40$ people), $C S=0 \%$ (0/37 people). More people were inclined to look at the AOI - the "old vault" as the nave got shorter visitors count AS-50\% (20/4people), BS-90\% (36/40people), CS-100\% (37/37people). The trends of the new and old research are similar.

Another value that might suggest lower cognitive interest is the time the participants spent looking at the floor. That area is the least diverse and almost entirely devoid of detail, so it seems hardly exciting and lacking in eye-catching irregularities ${ }^{53}$. Again, for the interior with the longest nave only 4 out of 37 people had at least one fixation within this AOI, whereas their number for the medium-length interior equaled 9 people out of 40 , and 12 out of 40 for the interior with the shortest nave. It is interesting that in the first study, an analogical tendency was observed - the longer the nave of the cathedral became, the more prone the participants were to ignore the floor entirely. This observation confirms the trends captured in the previous 2D eye-tracking research. The behavior of participants depicted above shows a distinct tendency in which the willingness to move one's head decreases along with the increasing length of the nave. Again, previous observation was confirmed.

After finishing the general analysis of the way the participants of the VR test looked at the fragments visible in the previous study, one should take a closer look at the individual characteristics of the particular AOls and compare them with the

Page $9 / 21$ 
tendencies observed when analyzing visual reactions to flat stimuli. An element that might have influenced the results of the VR research was physical discomfort - mostly neck fatigue ${ }^{54}$. - connected with the weight of the device the participants put on their heads. Participants in all three parts were equally exposed to this inconvenience. In case of the research discussed in this paper, approximately $1 / 4$ of the volunteers in each of the three groups mentioned this sort of discomfort. This is not the only research doubt regarding the use of the eye tracker in the VR environment ${ }^{55}$.

The data on the presbytery will be compared first. The longer the interior became in the images, the more observers had at least one fixation within the Presbytery $\mathrm{AOI}$ ( $A S=95 \%, B S=98 \%, C S=100 \%$ ). One reason why it is interesting is that the most distant presbytery - located at the end of the longest nave - obviously becomes the smallest of the three analyzed and yet it drew attention of the largest number of observers. In the test involving a stationary eye-tracker the number of visitors was not a feature suggesting variety, since only one person in the entire study had no recorder fixations within the Presbytery AOI. However, this tendency - visible in the results of the VR test - supports the theory of the longitudinal church effect. Similarly, the number of revisits within the Presbytery AOI in the original study did not vary a lot between the stimuli and did not show any specific tendency (the average range was from 2.6 to 2.9 revisits depending on the stimulus). However, in the VR study it was possible to observe a trend in which the lengthening of the nave resulted in fewer participants returning their gaze to the presbytery. Those exposed to the stimulus with the shortest nave usually had 2 to 4 revisits (2.85 revisits on average). The medium-length nave provoked 2 or 3 revisits (an average of 2.43), whereas almost everyone who looked at the image with the longest interior returned their gaze to the presbytery only twice (2.21 revisits on average). Such data, when presented separately from everything else might seem difficult to interpret and might even seem to contradict the idea of the longitudinal church effect. However, there is another possibility: the lower number of revisits might be due to the significant difference in the size of the AOI in question. The presbytery AOI in the stimulus AS is four times as large as in the stimulus CS, so it is only logical that people will return to it more often to look for more details. The average duration of a single fixation within the Presbytery $A O I$ increases with the length of the nave $(A S=0.169 \mathrm{~s} ; B S=$ $0.180 \mathrm{~s}, \mathrm{CS}=0.207 \mathrm{~s}$ ) (Fig. 5). A similar tendency was noticed in the research done on flat images in $2016(\mathrm{~A}=0.241 \mathrm{~s}, \mathrm{~B}=$ $0.312 \mathrm{~s}, \mathrm{C}=0.378 \mathrm{~s}$ ) even though the differences between the stimuli noticed in the current study were smaller in degree. Previously this tendency was interpreted as a feature testifying to the visual magnetism of the presbytery increasing with its getting further and further away from the observer. The test involving flat images showed no distinct relationship between the type of interior and the time of visual attention paid to the Presbytery AOI. The VR research showed a tendency contradictory to the hypothesis under verification - it turned out that the smaller and more distant the presbytery was in the image, the less time the participants spent on average looking at it ( $A S=1.15 \mathrm{~s} ; \mathrm{BS}=1.104 \mathrm{~s}, \mathrm{CS}=0.93 \mathrm{~s}$ ) (Fig. 6). When it comes to the time to first fixation of a participant is recorded within the Presbytery AOI, there is some discrepancy between the tendencies observed in the original test and those visible in the new experiment. It was those who were exposed to the stimulus BS, with the medium-length nave, that on average took interest in the presbytery the soonest - after $410 \mathrm{~ms}$. Those who saw the other two stimuli took much longer - on average it took them $1010 \mathrm{~ms}$ or $1450 \mathrm{~ms}$ for AS and CS respectively. In the new research, the way of supervising this aspect was, unfortunately, less precise and more vulnerable to errors and mistakes, which has been discussed earlier, in the supplementary materials describing the change in the way the images were switched. Like in previous research the role of the presbytery in shaping the funnel effect turned out to be difficult to assess. Among the five parameters, two would stimulate ritual focus, while two wouldn't and one remains uncertain.

A previous test revealed that the most important aspect of the interior perception is the vault ${ }^{22}$. This is why the nave cover which be discussed in detail. The registration done by means of a stationary eye tracker showed that the increasing length of the nave resulted in more and more people paying attention to the rhythm of the vaults leading to the presbytery. The analysis of the VR study also yielded interesting results as far as the perception of the vaults is concerned and allows to address the hypothesis based on the research ${ }^{45}$. Due to the small number of participants who looked at the vaults located above their vantage point or behind their symbolic back (Fig. 5), the analysis is restricted to the Old Vaults AOI. In the previous study, this element was found to be very significant cognitively. Great attention should be paid to determining

Page 10/21 
whether it fulfills such a role for 3D stimuli. This will allow one of the hypotheses to be verified ${ }^{45}$. Along with the lengthening of the nave the average interest in looking at the Old Vaults AOI visibly increased. For AS only $50 \%$ of observers looked at this part of the image. For the stimulus with the medium-length interior as many as $90 \%$ of the participants had a recorded fixation in this AOI, whereas for CS this number amounted to $98 \%$ - only one person out of 37 did not look at the Old Vaults AOI at all. The data processed after the previous study indicate that only the shortest nave and therefore, the shortest series of vaults - failed at drawing the attention of all participants (83\% did look at it), whereas for both the medium-length and the longest nave all the people exposed to these images had at least one recorded fixation within this AOI. The tendency observed in the VR research supports the interpretation presented in the paper describing the original test involving flat images. Another feature that might characterize the experience related to the lengthening of the vaults is the time that passed before the participants had their first fixation within the Old Vaults AOI. The data indicates that the time after which the volunteers usually began looking at this part of the interior got shorter as the length of the interior increased $(A S=4.57 \mathrm{~s}, B S=3.60 \mathrm{~s}, C S=2.74 \mathrm{~s})$. No similar relationship was observed in the previous test $(A=1.567$ $\mathrm{s}, \mathrm{B}=1.733 \mathrm{~s}, \mathrm{C}=0.987 \mathrm{~s}$ ). The time after which the participants first looked at the ceiling was significantly longer. This represents a decrease in visual magnetism observed in 3D versus 2D. The duration of an average fixation for the Old Vaults $\mathrm{AOI}$ increased slightly as the nave got longer ( $A S=0.112 \mathrm{~s}$; $B S=0.121 \mathrm{~s}, C S=0.133 \mathrm{~s}$ ) (Fig. 6). The same tendency was noticeable during the previous test, but the average duration of fixations within the Old Vaults AOI in the VR test proved to be significantly shorter $(A=0.166 \mathrm{~s}, B=0.240 \mathrm{~s}, C=0.241 \mathrm{~s})$. Another analyzed feature is the average time the participants spent looking at different spots within this particular $\mathrm{AOI}$. Dwell time gradually increased along with the lengthening of the interior. It is extremely interesting that this particular value in the VR test is astonishingly similar to the results obtained in the test involving flat images and a stationary eye tracker (Table S2). Such observation does not support the hypothesis according to which, after changing the way of presenting the stimulus to more active areas playing off important cognitive functions, they gain more attention.

\section{Conclusions And Recommendations}

A large portion of the newly-collected data indicates that the attention of observers was directed much more effectively towards the presbytery when the church's interior was longer. Only a few analyzed features, 3 out of 14 to be precise, (Table S3) do not support the author's hypothesis. What is important, the function of the presbytery itself seems of little importance when it comes to shaping this effect. Both tests show - but the VR one does it more vividly - that it is the vaults that have a dominating role in perceiving the depth of a church. That is why the author believes that future research in a field of art history and aesthetics should deal with Gothic interiors differing only in the type of vaults.

The characteristics discussed in the paper (number of fixations, number of visitors, dwell time) suggest that, even though a flat image is a major simplification of a real-life cognitive situation, the dominating reactions of the participants were fairly similar to those of the volunteers exposed to a more realistic experience of spherical images. Perhaps it will prove a rule for all symmetrical spaces characterized by a strongly directional, axial composition consisting of rhythmically repetitive elements. It is also possible that there is no need to employ VR technology for an eye-tracking interpretation of how different means of architectural expression are perceived when the most important elements of the composition are visible in a single image. Other assumptions in the introduction have also been confirmed. The areas generating the most visual attention as perceived in 3D remained so after flattening. These areas, as their appearance changed, were subject to corresponding modifications.

One of important change is fixational activity. In VR fixations are on average twice as fast as those recorded in the first study. This, together with the research carried out by other scientists ${ }^{17,18}$, allows us to conclude that there is a general tendency to shorten the duration of fixation during VR studies.

The experiment can be seen as a promising starting point for comparing aesthetic experience between VR and 2D eyetracking research. Despite the need for more comprehensive future studies, it proved that there is no need for scholars dealing with perception of architecture and heritage to stop using stationary eye trackers. The use of such a device has its

Page $11 / 21$ 
limits, but when appropriate methodology is applied and that data is collected and interpreted with care and precision, the results of such a test may be expected to be positively verified by a similar experiment in a 3D environment. Research done on photographs, and visualizations depicting historical monuments has every right to be continued. It is worth noticing that one research hypothesis hasn't been confirmed. Vaults presented at 3D stimulus in many aspects attracted active observers less than the vaults presented passively on a flat image. Many features have not changed.

Research involving 2D stimuli may seem attractive since the process of gathering eye-tracking information during the experiment in a VR environment is more difficult (see Comparison of eye trackers in the context of their usability) and has many limitations. Due to multiple problems, often problematic to identify, a large number of registrations were deemed flawed. This is why the group of people invited to the tests has to be considerably larger than the intended number of usable registrations - and in turn more money is needed to be used both as an incentive for the participants and for other purposes. Also, the analysis of the data collected from VR tests is much more complex and automatically more timeconsuming. And yet, even when modern technology was applied, some aspects of the interpretation simply did not provide clear, unambiguous results.

\section{Declarations}

\section{Acknowledgments}

\section{Author contributions}

Not applicable

Competing interests:

The author declares no competing interests.

\section{Data availability statement:}

The of datasets generated from 68 participants and analyzed during the current study are available in the RepOD repository. A lack of consent from all participants means other eye-tracking data are not publicly available. In line with the applicable legal and ethical principles, such a right belonged to volunteers. All graphic materials are included in the supplement. Study consent forms are preserved in the WUST archives.

Marta Rusnak, Problem of 2D representation of 3D objects in architectural and heritage studies. Re-analysis of phenomenon of longitudinal church, doi:10.18150/GMKZHI, RepOd V1; https://repod.icm.edu.pl/dataset.xhtml? persistentld=doi:10.18150/GMKZHI

Participants declarations and ethical approval:

The research was in accordance with the procedure, reported to the Dean, and authorities of the Faculty of Architecture, Wrocław University of Science and Technology. The following threats or inconveniences were reported to the Ethics Committee: temporary load on the head and neck (maximum 15 minutes), pressure on the nose, pressure on the ears, inconvenience while putting on the goggles, the possibility of fear, claustrophobia, dizziness. The participants while using VR were sitting there had no chance of falling. Informed consent was obtained from all participants. In the approved information form participants were informed of the possibility of withdrawing from the study at any time without giving reasons for their withdrawal. The participants of the study were over 18 years of age and were rewarded with a gift voucher. In line with these declarations, it was found that the research did not violate the applicable ethical regulations.

Funding: 
This work was supported by the National Science Center, Poland (NCN) under Miniatura Grant [2021/05/X/ST8/00595].

\section{Cooperation:}

Hardware consultation: Ewa Ramus (Neuro Device, Warszawa, Poland), graphic cooperation: Wojciech Fikus (Wroclaw University of Science and Technology, Poland), data collecting: Mateusz Rabiega (Wroclaw University of Science and Technology, Poland), Magdalena Nawrocka (Wroclaw University of Science and Technology, Poland), Małgorzata Cieślik (independent, Wrocław, Poland), Daria Kruczek (independent, London, UK)

The author would like to thank Ewa Ramus, Neuro Device and the technology company Tobii for the opportunity to develop an initial prototype of VR eye-tracking implementation. It is worth mentioning the support of Andrzej Żak.

\section{References}

1. Zeki, S., Bao, Y. \& Pöppel, E. Neuroaesthetics: The art, science, and brain triptych. (2020) doi:10.1002/pchj.383.

2. di Dio, C. \& Vittorio, G. Neuroaesthetics: a review. Current Opinion in Neurobiology 19 (6), 682-687 (2009). https://doi.org/10.1016/j.conb.2009.09.001.

3. Dieckie, G. Is psychology relevant to aesthetics? The Philosophical Review 71, 285-302 (1962). https://doi.org/10.2307/2183429.

4. Makin, A. The gap between aesthetic science and aesthetic experience. Journal of Counsciusness Studies 24 (1-2) 184-213 (2017).

5. Piotr Francuz. Imagia. Towards a neurocognitive image theory (KUL 2019).

6. Walker, F., Bucker, B. Anderson, N.C., Schreij, D. \& Theeuwes, J. Looking at paintings in the Vincent Van Gogh Museum: Eye movement patterns of children and adults. PLoS ONE 12, (2017). https://doi: 10.1371/journal.pone.0178912.

7. Mitrovic, A., Hegelmaier, L. M., Leder, H. \& Pelowski, M. Does beauty capture the eye, even if it's not (overtly) adaptive? A comparative eye-tracking study of spontaneous attention and visual preference with vast abstract art. Acta Psychologica 209, 103133 (2020). doi: 10.1016/j.actpsy.2020.103133.

8. Jankowski, T., Francuz, P., Oleś, P., Chmielnicka-Kuter, E. \& Augustynowicz, P. The Effect of Painting Beauty on Eye Movements. Advances in cognitive psychology 16, 213-227 (2020). https://doi: 10.5709/acp-0298-4.

9. Ferretti, G. \& Marchi, F. Visual attention in pictorial perception. Synthese 199, 2077-2101 (2021). https://doi.org/10.1007/s11229-020-02873-z.

10. Coburn, A., Vartanian, O. \& Chatterjee, A. Buildings, Beauty, and the Brain: A Neuroscience of Architectural Experience. Journal of Cognitive Neuroscience 29(9), 1521-1531 (2017). https://doi: 10.1162/jocn_a_01146.

11. Clay, V., König, P. \& König, S. U. Eye tracking in virtual reality. Journal of Eye Movement Research 12, (2019). https://doi.org/10.16910/jemr.12.1.3.

12. Brielmann, A. A., Buras, N. H., Salingaros, N. A. \& Taylor, R. P. What Happens in Your Brain When You Walk Down the Street? Implications of Architectural Proportions, Biophilia, and Fractal Geometry for Urban Science, Urban Science 6(1) (2022) doi:10.3390/urbansci6010003.

13. Salingaros, N. A. \& Sussman, A. Biometric Pilot-Studies Reveal the Arrangement and Shape of Windows on a Traditional Façade to be Implicitly “Engaging”, Whereas Contemporary Façades Are Not. Urban Science 4(26) (2020) doi:10.3390/urbansci4020026.

14. Lisińska-Kuśnierz, M. \& Krupa, M. P. Suitability of Eye Tracking in Assessing the Visual Perception of Architecture A Case Study Concerning Selected Projects Located in Cologne. Buildings 10(2) (2020). https://doi.org/10.3390/buildings10020020. 
15. Locher, P. J. Empirical investigation of the elements of composition in paintings. in Paintings as stimuli. The Cambridge handbook of the psychology of aesthetics and the arts, 221-242 (2014).

16. Vessel, E. A. \& Rubin, N. Beauty and the beholder: Highly individual taste for abstract, but not real-world images. Journal of Vision 10(18), (2010). https://doi: 10.1167/10.2.18.

17. Gulhan, D., Durant, S. \& Zanker, J. M. Similarity of gaze patterns across physical and virtual versions of an installation artwork. Scientific Reports 11, 18913 (2021). https://doi.org/10.1038/s41598-021-91904-x.

18. Dong, W. et al. Wayfinding Behavior and Spatial Knowledge Acquisition: Are They the Same in Virtual Reality and in Real-World Environments? Annals of the American Association of Geographers 112(1), 226-246 (2022). https://doi:10.1080/24694452.2021.1894088.

19. Panofsky, E. Architecture gothique et pensée scolastique précédé de L'abbé Suger de Saint-Denis, Les Edition de Minuit, Alençon. (Les Edition de Minuit, 1992).

20. Henry-Claude M., Stefanon L., Zaballos Y., Principes et éléments de l'architecture religieuse médievale (Fragile, 1997).

21. Scot, R.A. The Gothic Enterprise. Guide to understand the Medieval Cethedral. (University California Press 2003).

22. Rusnak, M. A., Fikus, W. \& Szewczyk. J., How do observers perceive the depth of a Gothic cathedral interior along with the change of its proportions? Eye tracking survey. Architectus, 53, 77-88 (2018). doi:10.5277/arc180105.

23. https://vr.tobii.com/

24. Liu, H., Laeng, B. \& Czajkowski, N. O. Does stereopsis improve face identification? A study using a virtual reality display with integrated eye-tracking and pupillometry. Acta Psychologica 210, 103142 (2020).

https://doi.org/10.1016/j.actpsy.2020.103142.

25. Bozkir, E. et al. Exploiting Object-of-Interest Information to Understand Attention in VR Classrooms. in 2021 IEEE Virtual Reality and 3D User Interfaces (VR) 597-605 (2021). doi:10.1109/VR50410.2021.00085.

26. Mirault, J., Guerre-Genton, A., Dufau, S. \& Grainger, J. Using virtual reality to study reading: An eye-tracking investigation of transposed-word effects. Methods in Psychology 3, 100029 (2020). doi:10.1109/VR.2019.8797896.

27. Xi, N. \& Hamari, J. Shopping in virtual reality: A literature review and future agenda. Journal of Business Research 134, 37-58 (2021). https://doi.org/10.1016/j.jbusres.2021.04.075.

28. Tian, P. et al. Behavior Analysis of Indoor Escape Route-Finding Based on Head-Mounted VR and Eye Tracking. in 2019 International Conference on Internet of Things (iThings) and IEEE Green Computing and Communications (GreenCom) and IEEE Cyber, Physical and Social Computing (CPSCom) and IEEE Smart Data (SmartData) 422-427 (2019). doi:10.1109/iThings/GreenCom/CPSCom/SmartData.2019.00090.

29. Chihming, W., Zexin, J., Yuxin, L., Songqing, H. \& Zhongwei, Y. Investigation on the Eye-tracking Technology in Hazard Identification of Building Construction Engineering. in 2020 IEEE 2nd International Conference on Architecture, Construction, Environment and Hydraulics 32-35 (ICACEH, 2020). doi:10.1109/ICACEH51803.2020.9366265.

30. Zhang, L., Jeng, T. \& Zhang, R.-X. Integration of virtual reality, 3-D eye-tracking, and protocol analysis for re-designing street space. in Alhadidi, S., Crolla, K., Huang, W., Janssen, P., \& Fukuda, T. (Eds.), CAADRIA 2018-23rd International Conference on Computer-Aided Architectural Design Research in Asia: Learning, Prototyping and Adapting $<\mathrm{bi}>1(1)$ $</$ bi $>$, 431-440 (CAADRIA 2018).

31. Zhang, R. X. \& Zhang, L. M. Panoramic visual perception and identification of architectural cityscape elements in a virtual-reality environment. Future Generation Computer Systems 118, 107-117 (2021).

32. Zhang, L.-M., Zhang, R.-X., Jeng, T.-S. \& Zeng, Z.-Y. Cityscape protection using VR and eye tracking technology. Journal of Visual Communication and Image Representation 64, 102639 (2019).

33. Mokatren, M., Kuflik, T. \& Shimshoni, I. Exploring the potential of a mobile eye tracker as an intuitive indoor pointing device: A case study in cultural heritage. Future Generation Computer Systems 81, 528-541 (2018). 
34. Jung, Y. J., Zimmerman, H. T. \& Pérez-Edgar, K. A Methodological Case Study with Mobile Eye-Tracking of Child Interaction in a Science Museum. TechTrends 62, 509-517 (2018).

35. Rusnak, M. \& Szewczyk, J. Eye tracker as innovative conservation tool. Ideas for expanding range of research related to architectural and urban heritage. 25-35. Journal of Heritage Conservation 54, 25-35 (2018). doi:10.17425/WK54EYETRACK.

36. Rusnak, M. \& Ramus, E. With an eye tracker at the Warsaw Rising Museum: valorization of adaptation of historical interiors. Journal of Heritage Conservation 58, 78-90 (2019). doi:10.17425/WK58WARSAW.

37. Junker D, N. Ch. Mobile eyetracking in landscape architecture. Analysing behaviours and interactions in natural environments by the use of innovative visualizations. in Proceeding of The International Conference "Between Data and Science" Architecture, neuroscience and the digital worlds. (2018). doi:10.5772/intechopen.74992.

38. Kabaja B, K. M. Possibilities of using the eye tracking method for research on the historic architectonic space in the context of its perception by users (on the example of Rabka-Zdrój). Part 1. Preliminary remarks. Joural of Heritage Conservation 52, 74-85 (2017). doi:10.17425/WK52EYETRACK

39. Kiefer, P., Giannopoulos, I., Kremer, D., Schlieder, C. \& Martin, R. Starting to get bored: An outdoor eye tracking study of tourists exploring a city. in Eye Tracking Research and Applications Symposium (ETRA) (2014). doi:10.1145/2578153.2578216.

40. Karakas, T. \& Yildiz, D. Exploring the influence of the built environment on human experience through a neuroscience approach: A systematic review. Frontiers of Architectural Research 9, 236-247 (2020).

https://doi.org/10.1016/j.foar.2019.10.005.

41. Mohammadpour, A. Karan, E., Asadi, S. \& Rothrock L. Measuring End-User Satisfaction in the Design of Building Projects Using Eye-Tracking Technology. Computing in Civil Engeniering 2015 (2015) https://doi.org/10.1061/9780784479247.070.

42. S. Białowąs, A. S. Eye-tracking in Marketing Reaserch. in Managing Economic Innovations - Methods and Instruments 91-104 (Bogucki Wydawnictwo Naukowe 2019).

43. Dupont, L., Ooms, K., Duchowski, A. T., Antrop, M. \& Eetvelde, V. van. Investigating the visual exploration of the ruralurban gradient using eye-tracking. Spatial Cognition \& Computation 17, 65-88 (2017).

https://10.1080/13875868.2016.1226837.

44. Holmqvist, K., Nyström, M. \& Mulvey, F. Eye Tracker Data Quality: What It is and How to Measure It. in Proceedings of the Symposium on Eye Tracking Research and Applications 45-52 (Association for Computing Machinery, 2012). doi:10.1145/2168556.2168563.

45. Foulsham, T., Walker, E. \& Kingstone, A. The where, what and when of gaze allocation in the lab and the natural environment. Vision Research 51 (17), 1920-1931 (2011). https://doi.org/10.1016/j.visres.2011.07.002.

46. van Herpen, E., van den Broek, E., van Trijp, H. C. M. \& Yu, T. Can a virtual supermarket bring realism into the lab? Comparing shopping behavior using virtual and pictorial store representations to behavior in a physical store. Appetite 107 (2016) doi:10.1016/j.appet.2016.07.033.

47. Haskins, A. J., Mentch, J., Botch, T. L. \& Robertson, C. E. Active vision in immersive, $360^{\circ}$ real-world environments. Sceintific reports 10, 14304 (2020). https://doi.org/10.1038/s41598-020-71125-

48. https://www.tobiipro.com/.

49. Pangilinan, E., Lukas, S. \& Mohan V. Creating Augmented and Virtual Realities: Theory and Practice for NextGeneration Spatial Computing. (O’Reilly Media, 2019).

50. Tatler, B. W., Wade, N. J., Kwan, H., Findlay, J. M. \& Velichkovsky, B. M. Yarbus, eye movements, and vision. i-Perception 1(1) (2010). doi:10.1068/i0382.

51. Rusnak, M. Problem of 2D representation of 3D objects in architectural and heritage studies. Re-analysis of phenomenon of longitudinal church. RepOp V1, (2021). https://doi.org/10.18150/GMKZHI. 
52. Galley. N., D. B. and C. B. Fixation Durations - Why Are They So Highly Variable?. in Advances in Visual Perception Research 83-106 (Nova Biomedicaal, 2015). https://doi:10.13140/RG.2.1.3128.1769.

53. Groner, R. \& Groner, M. T. Attention and eye movement control: An overview. European archives of psychiatry and neurological sciences 239, 9-16 (1989). https://doi.org/10.1007/BF01739737.

54. McGill, M., Kehoe, A., Freeman, E. \& Brewster, S. A. Expanding the Bounds of Seated Virtual Workspaces. ACM Transactions on Computer-Human Interaction (TOCHI) 27, 1-40 (2020). https://doi.org/10.1145/3380959.

55. Mon-Williams M, Plooy A, Burgess-Limerick R \& Wann J. Gaze angle: a possible mechanism of visual stress in virtual reality headsets. Ergonomics 41, 280-285 (1998). doi:10.1080/001401398187035.

\section{Figures}
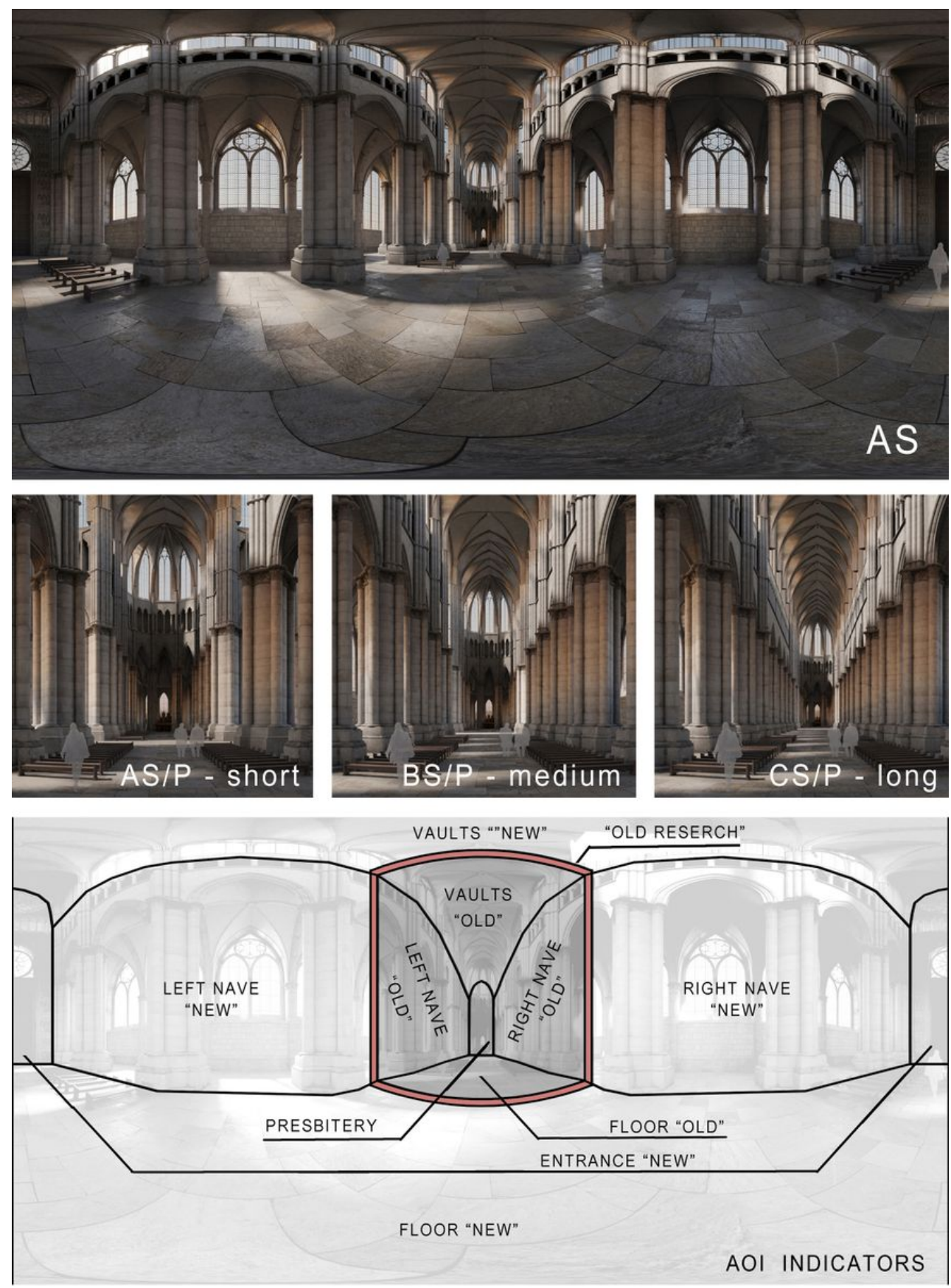

Figure 1 
Prepared spherical visualizations and division into AOI. AS - flattened spherical image prepared for the cathedral with a short nave, AS/P - fragment of the spherical image for the cathedral with a short nave, which presents a part of the nave and the presbytery, BS/P - fragment of the spherical image for the cathedral with a medium-length nave, which presents a part of the nave and the presbytery, CS/P - fragment of the spherical image for the cathedral with a long nave, which presents a part of the nave and the presbytery, AOI INDICATORS - the manner of allocating and naming the Areas of Interest (Marta Rusnak on the basis of visualization done by Wojciech Fikus)

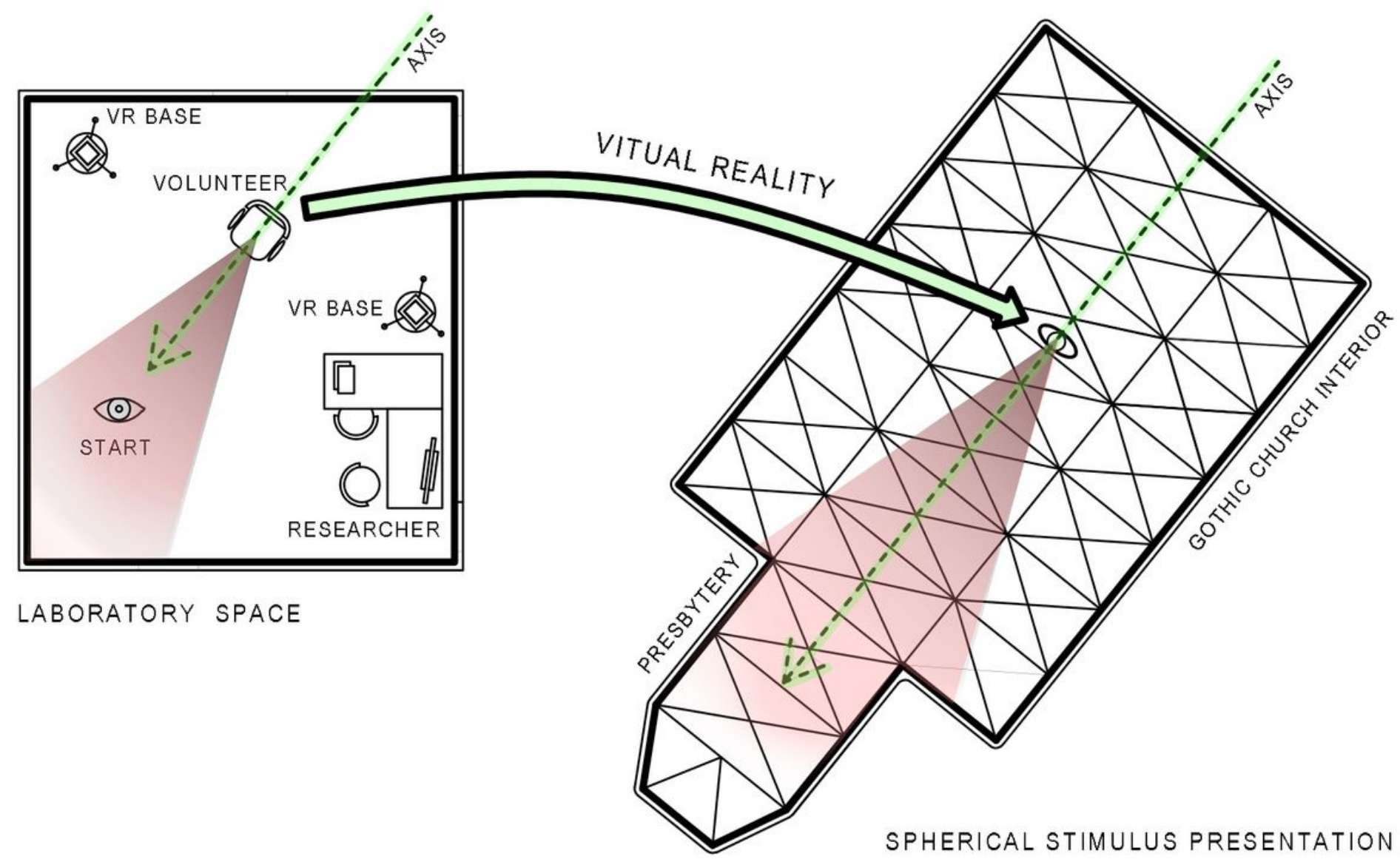

Figure 2

Location of equipment in the lab. The idea was to maintain consistency between the lab space and the way spherical stimuli were presented. 


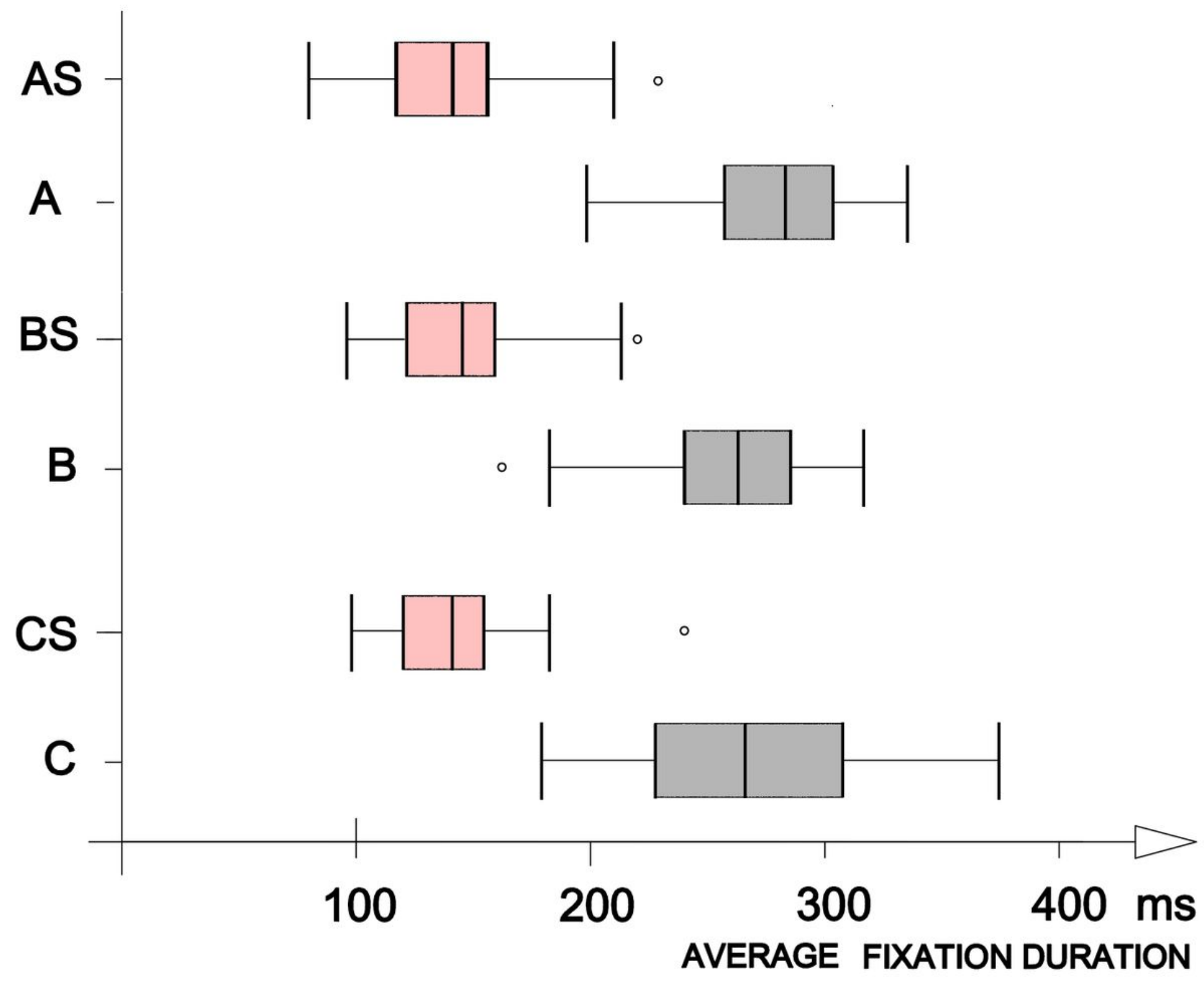

Figure 3

Comparison of the average fixation duration for the tested examples. Box plots for 2D stimuli are marked on pink and 3D for stimuli are grey. 


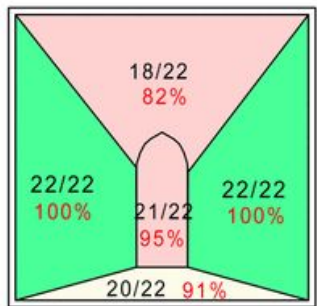

SHORT NAVE - A

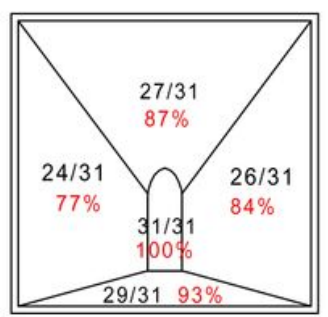

MEDIUM NAVE - B

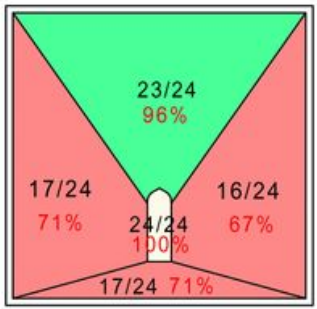

LONG NAVE - C

2D FLAT STIMULI
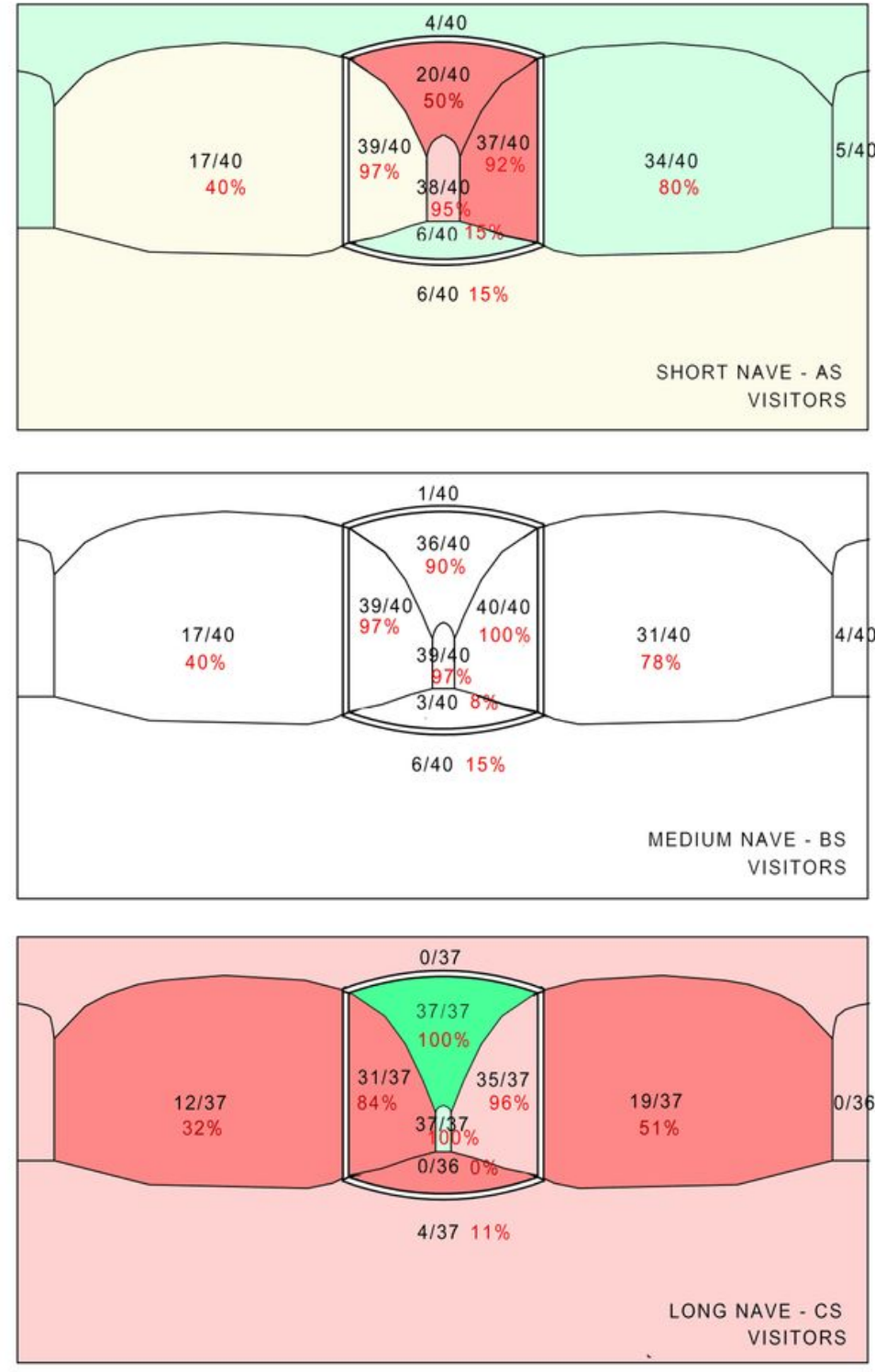

3D SPHERICAL STIMULI
LEGEND

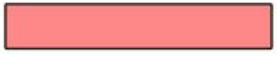

SIGNIFICANTLY SMALLER

PARAMETER

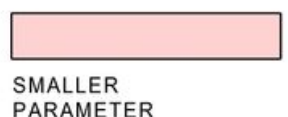

PARAMETER

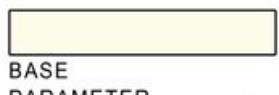

PARAMETER

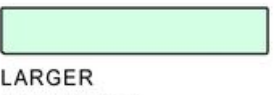

PARAMETER

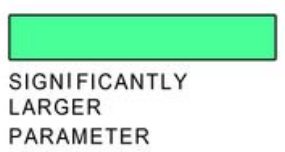

\section{Figure 4}

Visitors count - comparison between interiors with short, medium-length and long naves for 2D and 3D stimuli. In order to make the interpretation easier, the interior with the medium-length nave serves as point of reference. 

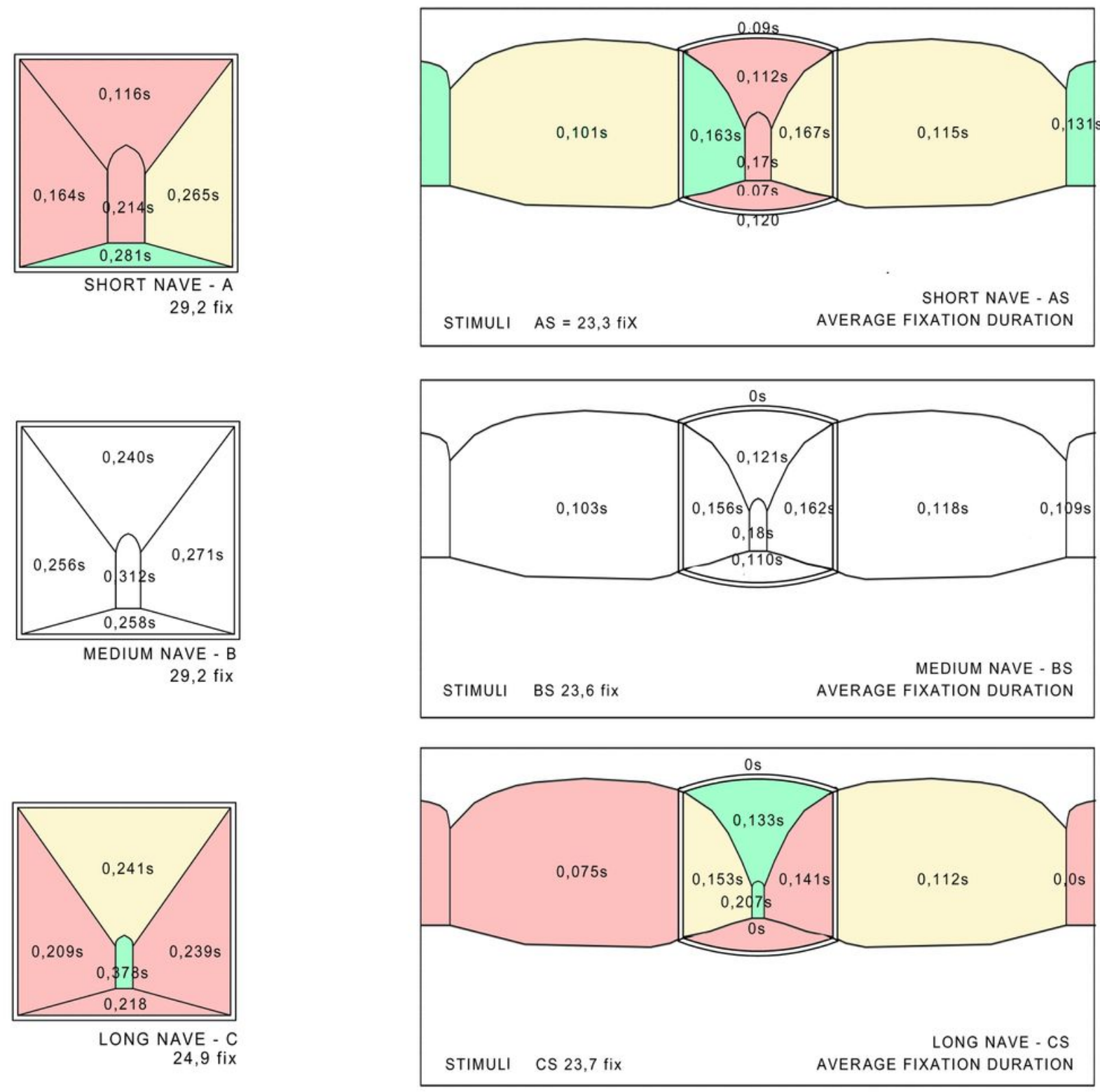

LEGEND

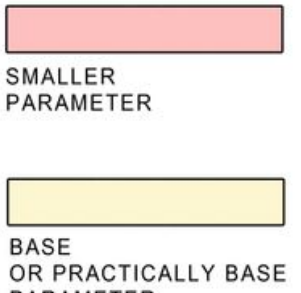

PARAMETER

\section{D FLAT STIMULI}

\section{Figure 5}

Average fixation duration - comparison between interiors with short, medium-length and long naves. In order to make the interpretation easier, the interior with the medium-length nave serves as point of reference. 
OUTSIDE THE PICTURE $=5,1 \%$

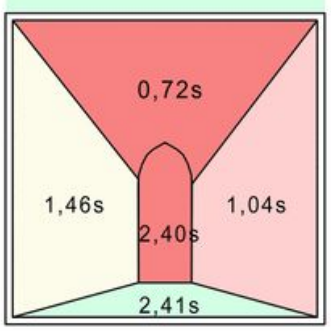

SHORT NAVE - A

TOTAL VISIT DURATION

OUTSIDE THE PICTURE $=3,2 \%$

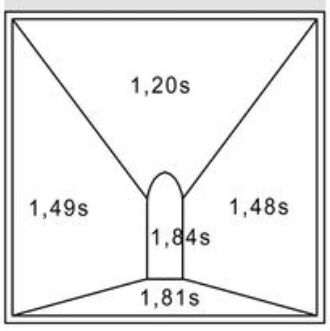

MEDIUM NAVE - B TOTAL VISIT DURATION

OUTSIDE THE PICTURE $=1,2 \%$

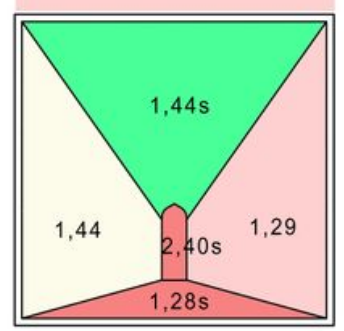

LONG NAVE - C

TOTAL VISIT DURATION

2D FLAT STIMULI
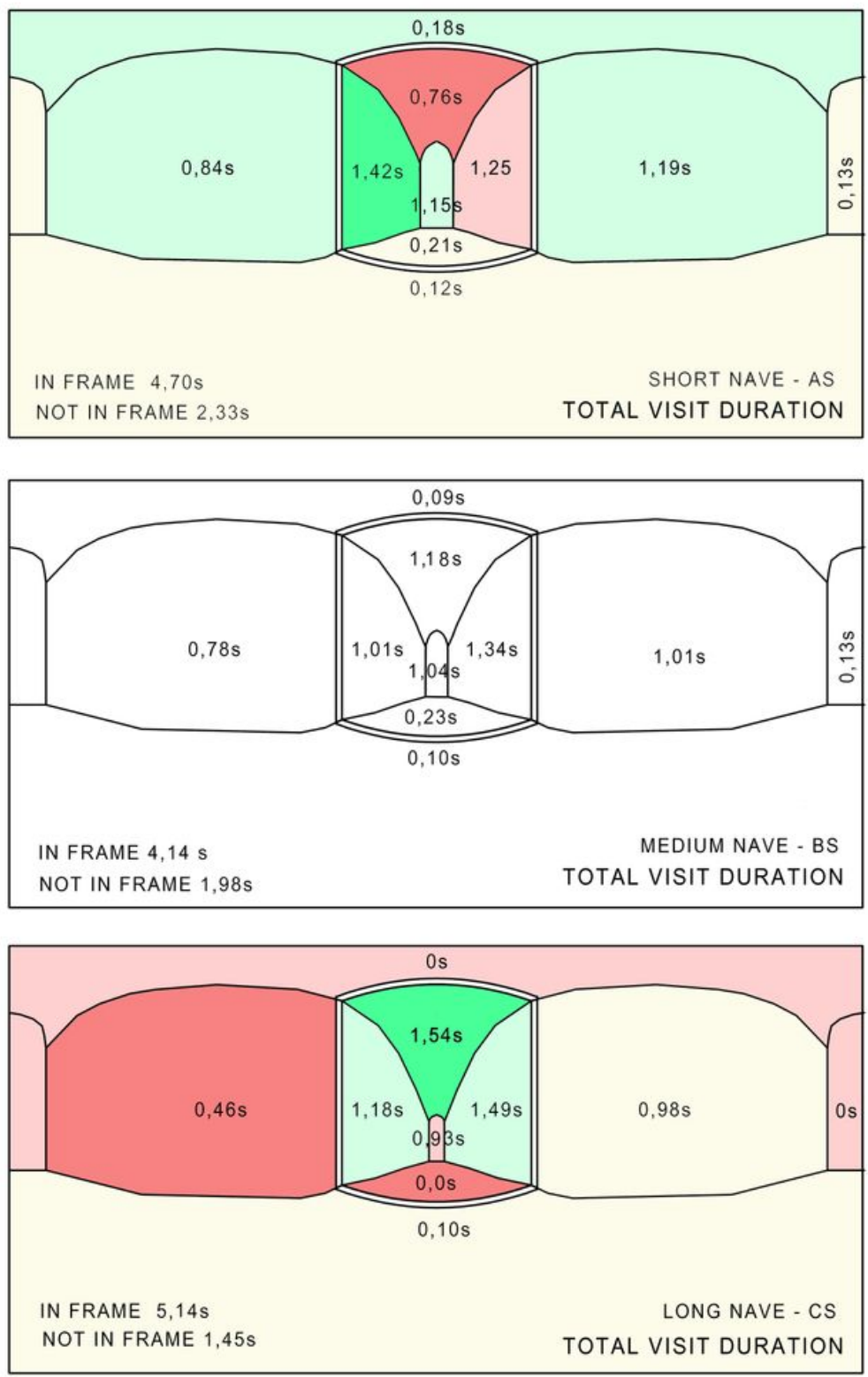

OR PRACTICALLY BASE PARAMETER

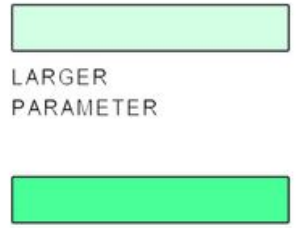

SIGNIFICANTLY

LARGER

PARAMETER

\section{Figure 6}

Total visit duration - comparison between three interiors. In order to make the interpretation easier, the interior with the medium-length nave serves as point of reference.

\section{Supplementary Files}

This is a list of supplementary files associated with this preprint. Click to download.

- 24022022CHALLENGE2Dvs3Dsupplementarymatherials.docx 